\title{
Construction of exact solutions for fractional-type difference-differential equations via symbolic computation
}

\author{
İsmail Aslan* \\ Department of Mathematics, Izmir Institute of Technology, Urla, İzmir 35430, Turkey
}

\section{A R T I C L E I N F O}

\section{Article history:}

Received 5 June 2013

Received in revised form 8 July 2013

Accepted 10 July 2013

Available online 17 July 2013

\section{Keywords:}

Difference-differential equation

Lattice equation

Extended simplest equation method

\begin{abstract}
A B S T R A C T
This paper deals with fractional-type difference-differential equations by means of the extended simplest equation method. First, an equation related to the discrete KdV equation is considered. Second, a system related to the well-known self-dual network equations through a real discrete Miura transformation is analyzed. As a consequence, three types of exact solutions (with the aid of symbolic computation) emerged; hyperbolic, trigonometric and rational which have not been reported before. Our results could be used as a starting point for numerical procedures as well.
\end{abstract}

(c) 2013 Elsevier Ltd. All rights reserved.

\section{Introduction}

The appearance of difference-differential equations (DDEs) or lattice equations in nature is quite common. They model many physical and engineering problems such as wave phenomena in fluids, pulses in biological chains, currents in electrical networks, particle vibrations in lattices, chemical reactions, and optical fibers. Their important role has motivated investigators to develop a number of integrable DDEs since the original work of Fermi, Pasta and Ulam [1]. To name a few; Volterra lattice equation [2], discrete KdV equation [3], Toda lattice equation [4], Ablowitz-Ladik lattice equation [5], discrete sine-Gordon equation [6], discrete modified KdV equation [7], see [8] for a list of DDEs. These DDEs are of (or can be converted to) the form $\dot{u}_{n}=P\left(\ldots, u_{n-1}, u_{n}, u_{n+1}, \ldots\right)$, where $P$ is a polynomial of its arguments. Unlike difference equations which are completely discretized, DDEs are semi-discretized with some (or all) of their space variables discretized while time is usually kept continuous. For this reason, they can be thought as hybrid equations. Apart from their physical relevance, DDEs also play a crucial role in numerical simulations of nonlinear partial differential equations.

In this study, our attention is focused towards fractional-type DDEs of the form

$\dot{u}_{n}=R\left(\ldots, u_{n-1}, u_{n}, u_{n+1}, \ldots\right)$

where $u_{n}(t)=u(n, t)$ the displacement of the $n$th particle from the equilibrium position and $n \in \mathbb{Z}$. Eq. (1) is called fractional-type in

\footnotetext{
* Tel.: +90 232750 7765; fax: +90 2327507777 .

E-mail address: ismailaslan@iyte.edu.tr
}

the sense that $R$ is a rational function of its arguments. First, we consider the integrable equation $[9,10]$

$\dot{u}_{n}=\frac{u_{n-1}-u_{n+1}}{1+u_{n-1}-u_{n+1}}$,

from which the discrete $\mathrm{KdV}$ equation can be directly produced [11]. Second, our target will be the system [12]

$\dot{u}_{n}=\frac{\left(v_{n+1}-v_{n-1}\right)\left(1-u_{n}^{2}\right)\left(1-v_{n}^{2}\right)}{\left(v_{n-1}+v_{n}\right)\left(v_{n}+v_{n+1}\right)}$,
$\dot{v}_{n}=\frac{\left(u_{n+1}-u_{n-1}\right)\left(1-u_{n}^{2}\right)\left(1-v_{n}^{2}\right)}{\left(u_{n-1}+u_{n}\right)\left(u_{n}+u_{n+1}\right)}$,

which is related to the self-dual network equations [13]

$\dot{V}_{m}=\left(I_{m}-I_{m+1}\right)\left(1-V_{m}^{2}\right)$,
$\dot{I}_{m}=\left(V_{m-1}-V_{m}\right)\left(1-I_{m}^{2}\right)$,

via the real discrete Miura transformation

$m=n / 2$,

$V_{m}=\frac{1+u_{n+1 / 2} u_{n+3 / 2}}{u_{n+1 / 2}+u_{n+3 / 2}}$

$I_{m}=\frac{1+v_{n-1 / 2} v_{n+1 / 2}}{v_{n-1 / 2}+v_{n+1 / 2}}$.

Indeed, the extension (3) is quite natural and useful because Eq. (4) has been explored extensively in works $[14,15]$. As far as we could verify, relatively less work is being performed for the symbolic computation of exact solutions to fractional-type DDEs while there has 
been a considerable amount of work done in finding exact solutions to polynomial DDEs.

In the last decade, due to the increased interest in DDEs, a whole range of analytical solution methods such as Hirota's bilinear method [16], ADM-Padé technique [17], Casoratian technique [18], homotopy perturbation method [19], Exp-function method [20], and so on. were developed by the researchers. Numerous DDEs have been already solved by the just-mentioned methods and this number is still increasing due to some generalizations. However, most of the methods are not easy to handle or may not work depending on the problem under study. Nowadays, due to the increasing availability of technology, many of the methods take advantage of the availability of symbolic computation systems (such as MATHEMATICA, MAPLE and MATLAB), which avoid the need for performing the complex and tedious calculations "by hand" and eliminate error.

On the other hand, generalization of innovative approaches to the investigation of DDEs for traveling wave solutions and finding new results seems interesting and helpful to the reader in the scientific communities. Quite recently, traveling wave solutions of some complicated nonlinear evolution equations were successfully found with the aid of the extended simplest equation method [21]. This technique is based on a priori assumption that the solutions of a nonlinear ODE can be written in terms of some special functions which satisfy some ordinary differential equations referred to as the simplest equations. The simplest equation has two main features: first, it is the equation of a higher order than the nonlinear ODE to be solved; second, the general solution of this equation is known. In this work, we prefer to handle our Eqs. (2) and (3) by the extended simplest equation method. The adaptation of this method to fractional type DDEs can be summarized as follows [22].

\section{Methodology}

Consider a system of $M$ fractional type DDEs in the form

$R\left(\mathbf{v}_{\mathbf{n}+\mathbf{p}_{1}}(\mathbf{x}), \ldots, \mathbf{v}_{\mathbf{n}+\mathbf{p}_{k}}(\mathbf{x}), \ldots, \mathbf{v}_{\mathbf{n}+\mathbf{p}_{1}}^{\prime}(\mathbf{x}), \ldots, \mathbf{v}_{\mathbf{n}+\mathbf{p}_{k}}^{\prime}(\mathbf{x}), \ldots, \mathbf{v}_{\mathbf{n}+\mathbf{p}_{1}}^{(r)}(\mathbf{x}), \ldots, \mathbf{v}_{\mathbf{n}+\mathbf{p}_{k}}^{(r)}(\mathbf{x})\right)=0$,

where the dependent variable $\mathbf{v}_{\mathbf{n}}$ have $M$ components $v_{i, \mathbf{n}}$ and so do its shifts; the continuous variable $\mathbf{x}$ has $N$ components $x_{i}$; the discrete variable $\mathbf{n}$ has $Q$ components $n_{j}$; the $k$ shift vectors $\mathbf{p}_{i} \in \mathbb{Z}^{Q}$; and $\mathbf{v}^{(r)}(\mathbf{x})$ denotes the collection of mixed derivative terms of order $r$. To search for exact solutions of Eq. (6), we first take the wave transformation

$$
\begin{aligned}
\mathbf{V}_{\mathbf{n}+\mathbf{p}_{s}}(\mathbf{x})= & \mathbf{V}_{\mathbf{n}+\mathbf{p}_{s}}\left(\xi_{\mathbf{n}}\right), \quad \xi_{\mathbf{n}}=\sum_{i=1}^{Q} d_{i} n_{i}+\sum_{j=1}^{N} c_{j} x_{j}+\zeta, \\
& (s=1,2, \ldots, k),
\end{aligned}
$$

into consideration where the coefficients $c_{1}, c_{2}, \ldots, c_{N}, d_{1}, d_{2}, \ldots, d_{Q}$ and the phase $\zeta$ are all constants. Then, Eq. (6) changes into

$$
\begin{aligned}
& R\left(\mathbf{V}_{\mathbf{n}+\mathbf{p}_{1}}\left(\xi_{\mathbf{n}}\right), \ldots, \mathbf{V}_{\mathbf{n}+\mathbf{p}_{k}}\left(\xi_{\mathbf{n}}\right), \ldots, \mathbf{V}_{\mathbf{n}+\mathbf{p}_{1}}^{\prime}\left(\xi_{\mathbf{n}}\right), \ldots, \mathbf{V}_{\mathbf{n}+\mathbf{p}_{k}}^{\prime}\left(\xi_{\mathbf{n}}\right), \ldots,\right. \\
& \left.\quad \mathbf{V}_{\mathbf{n}+\mathbf{p}_{1}}^{(r)}\left(\xi_{\mathbf{n}}\right), \ldots, \mathbf{V}_{\mathbf{n}+\mathbf{p}_{k}}^{(r)}\left(\xi_{\mathbf{n}}\right)\right)=0 .
\end{aligned}
$$

To obtain an exact solution, a finite expansion in $\frac{\psi^{\prime}\left(\breve{n}_{\mathbf{n}}\right)}{\psi\left(\xi_{\mathbf{n}}\right)}$ like

$\mathbf{V}_{\mathbf{n}}\left(\xi_{\mathbf{n}}\right)=\sum_{l=0}^{m} a_{l}\left(\frac{\psi^{\prime}\left(\xi_{\mathbf{n}}\right)}{\psi\left(\xi_{\mathbf{n}}\right)}\right)^{l}$

is proposed as a (possible) solution of the nonlinear equation under study, where $m$ is a positive integer, $a_{i}$ 's are constants to be determined, $\psi\left(\xi_{\mathbf{n}}\right)$ is the general solution of the simplest equation which can be taken as

$\psi^{\prime \prime}\left(\xi_{\mathbf{n}}\right)+\mu \psi\left(\xi_{\mathbf{n}}\right)=0$, where $\mu$ is an arbitrary constant and prime denotes derivative with respect to $\xi_{\mathbf{n}}$. The general solution of Eq. (10) is well known to us. Thus, we have the following cases:

$\frac{\psi^{\prime}\left(\xi_{\mathbf{n}}\right)}{\psi\left(\xi_{\mathbf{n}}\right)}=\sqrt{-\mu}\left(\frac{C_{1} \cosh \left(\sqrt{-\mu} \xi_{\mathbf{n}}\right)+C_{2} \sinh \left(\sqrt{-\mu} \xi_{\mathbf{n}}\right)}{C_{1} \sinh \left(\sqrt{-\mu} \xi_{\mathbf{n}}\right)+C_{2} \cosh \left(\sqrt{-\mu} \xi_{\mathbf{n}}\right)}\right), \mu<0$,

$\frac{\psi^{\prime}\left(\xi_{\mathbf{n}}\right)}{\psi\left(\xi_{\mathbf{n}}\right)}=\sqrt{\mu}\left(\frac{-C_{1} \sin \left(\sqrt{\mu} \xi_{\mathbf{n}}\right)+C_{2} \cos \left(\sqrt{\mu} \xi_{\mathbf{n}}\right)}{C_{1} \cos \left(\sqrt{\mu} \xi_{\mathbf{n}}\right)+C_{2} \sin \left(\sqrt{\mu} \xi_{\mathbf{n}}\right)}\right), \mu>0$,

$\frac{\psi^{\prime}\left(\xi_{\mathbf{n}}\right)}{\psi\left(\xi_{\mathbf{n}}\right)}=\frac{C_{1}}{C_{1} \xi_{\mathbf{n}}+C_{2}}, \mu=0$,

where $C_{1}$ and $C_{2}$ are arbitrary constants. By a straightforward calculation, one can get the identity

$\xi_{\mathbf{n}+\mathbf{p}_{s}}=\xi_{\mathbf{n}}+\varphi_{s}, \quad \varphi_{s}=p_{s 1} d_{1}+p_{s 2} d_{2}+\cdots+p_{s Q} d_{Q}$,

where $p_{s j}$ is the $j$ th component of the shift vector $\mathbf{p}_{s}$. Hence, considering the trigonometric/hyperbolic function identities and using the functions (11a)-(11c) as well as (12), we derive the uniform shift formulas

$$
\begin{aligned}
& \mathbf{V}_{\mathbf{n}+\mathbf{p}_{s}}\left(\xi_{\mathbf{n}}\right)=\sum_{l=0}^{m} a_{l}\left(\frac{\frac{\psi^{\prime}\left(\xi_{\mathbf{n}}\right)}{\psi\left(\xi_{\mathbf{n}}\right)} \pm \sqrt{-\mu} \tanh \left(\sqrt{-\mu} \varphi_{s}\right)}{1 \pm \frac{1}{\sqrt{-\mu}} \tanh \left(\sqrt{-\mu} \varphi_{s}\right) \frac{\psi^{\prime}\left(\xi_{\mathbf{n}}\right)}{\psi\left(\xi_{\mathbf{n}}\right)}}\right)^{l}, \quad \mu<0, \\
& \mathbf{V}_{\mathbf{n}+\mathbf{p}_{s}}\left(\xi_{\mathbf{n}}\right)=\sum_{l=0}^{m} a_{l}\left(\frac{\frac{\psi^{\prime}\left(\xi_{\mathbf{n}}\right)}{\psi\left(\xi_{\mathbf{n}}\right)} \mp \sqrt{\mu} \tan \left(\sqrt{\mu} \varphi_{s}\right)}{1 \pm \frac{1}{\sqrt{\mu}} \tan \left(\sqrt{\mu} \varphi_{s}\right) \frac{\psi^{\prime}\left(\xi_{\mathbf{n}}\right)}{\psi\left(\xi_{\mathbf{n}}\right)}}\right)^{l}, \quad \mu>0, \\
& \mathbf{V}_{\mathbf{n}+\mathbf{p}_{s}}\left(\xi_{\mathbf{n}}\right)=\sum_{l=0}^{m} a_{l}\left(\frac{\frac{\psi^{\prime}\left(\xi_{\mathbf{n}}\right)}{\psi\left(\xi_{\mathbf{n}}\right)}}{1 \pm \varphi_{s} \frac{\psi^{\prime}\left(\xi_{\mathbf{n}}\right)}{\psi\left(\xi_{\mathbf{n}}\right)}}\right)^{l}, \quad \mu=0 .
\end{aligned}
$$

Balancing the highest-order derivative term and the highest order nonlinear term(s) in $\mathbf{V}_{\mathbf{n}}\left(\xi_{\mathbf{n}}\right)$ as in the continuous case, the degree $m$ of (9) and (13a)-(13c) from (8) can be easily determined. Because $\mathbf{V}_{\mathbf{n}+\mathbf{p}_{s}}$ can be thought as being of degree zero in $\frac{\psi^{\prime}\left(\tilde{n}_{\mathbf{n}}\right)}{\psi\left(\xi_{\mathbf{n}}\right)}$, the leading terms of $\mathbf{V}_{\mathbf{n}+\mathbf{p}_{s}}\left(\mathbf{p}_{s} \neq \mathbf{0}\right)$ will not have any effect on the balancing procedure. Substituting (9) and (13a)-(13c) together with (10) into (8), equating the coefficients of $\left(\frac{\psi^{\prime}\left(\xi_{\mathbf{n}}\right)}{\psi\left(\xi_{\mathbf{n}}\right)}\right)^{l}(l=0,1,2, \ldots)$ to zero, we obtain a system of nonlinear algebraic equations from which the undetermined constants $a_{i}, d_{i}, c_{j}$, and $k$ can be explicitly found. Finally, substituting these results into (9), one can derive varies kind of exact solutions to (6).

\section{Exact solutions for Eq. (2)}

To find traveling wave solutions of Eq. (2), we first introduce the transformation

$u_{n}=U_{n}\left(\xi_{n}\right), \quad \xi_{n}=d n+k t+\alpha$,

where $d$ and $k$ are real parameters to be specified, while $\alpha$ denotes the phase shift. Substituting (14) into Eq. (2) gives

$k U_{n}^{\prime}=\frac{U_{n-1}-U_{n+1}}{1+U_{n-1}-U_{n+1}}$,

where prime denotes derivative with respect to the new independent variable $\xi_{n}$. Our procedure suggests then to look for special solutions of (15) in the form

$U_{n}=a_{0}+a_{1}\left(\frac{\psi^{\prime}\left(\xi_{n}\right)}{\psi\left(\xi_{n}\right)}\right), \quad a_{1} \neq 0$

where $\psi\left(\xi_{n}\right)$ satisfies Eq. (10), while $a_{0}$ and $a_{1}$ are arbitrary constants to be determined at the stage of solving the problem. 


\subsection{Hyperbolic function solutions}

In case $\mu<0$, we first derive the expressions

$U_{n \pm 1}=a_{0}+a_{1}\left(\frac{\frac{\psi^{\prime}\left(\xi_{\mathbf{n}}\right)}{\psi\left(\xi_{\mathbf{n}}\right)} \pm \sqrt{-\mu} \tanh (d \sqrt{-\mu})}{1 \pm \frac{1}{\sqrt{-\mu}} \frac{\psi^{\prime}\left(\xi_{\mathbf{n}}\right)}{\psi\left(\xi_{\mathbf{n}}\right)} \tanh (d \sqrt{-\mu})}\right)$.

in accordance with (13a). Substituting (16) and (17) along with (10) into Eq. (15), clearing the denominator, setting the coefficients of $\left(\frac{\psi^{\prime}}{\psi}\right)^{l}(l=0,2,4)$ to zero, we derive a system of nonlinear algebraic equations for $a_{0}, a_{1}, d, k$ and $\mu$. Solving the resulting system, we get the relation

$a_{0}=a_{0}, \quad a_{1}=\frac{\tanh (d \sqrt{-\mu})}{2 \sqrt{-\mu}}, \quad k=-\frac{\sinh (2 d \sqrt{-\mu})}{\sqrt{-\mu}}$,

which yields a discrete hyperbolic function solution to Eq. (2) as

$$
\begin{aligned}
u_{n}(t)= & a_{0}+\frac{1}{2} \\
& \times \tanh (d \sqrt{-\mu})\left(\frac{C_{1} \cosh \left(\sqrt{-\mu} \xi_{n}\right)+C_{2} \sinh \left(\sqrt{-\mu} \xi_{n}\right)}{C_{1} \sinh \left(\sqrt{-\mu} \xi_{n}\right)+C_{2} \cosh \left(\sqrt{-\mu} \xi_{n}\right)}\right),
\end{aligned}
$$

where $\xi_{n}=d n-\frac{\sinh (2 d \sqrt{-\mu})}{\sqrt{-\mu}} t+\alpha$, while $a_{0}, d, \alpha, \mu(<0), C_{1}$ and $C_{2}$ remain arbitrary.

\subsection{Trigonometric function solutions}

In case $\mu>0$, we first derive the expressions

$U_{n \pm 1}=a_{0}+a_{1}\left(\frac{\frac{\psi^{\prime}\left(\xi_{\mathbf{n}}\right)}{\psi\left(\xi_{\mathbf{n}}\right)} \mp \sqrt{\mu} \tan (d \sqrt{\mu})}{1 \pm \frac{1}{\sqrt{\mu}} \frac{\psi^{\prime}\left(\xi_{\mathbf{n}}\right)}{\psi\left(\xi_{\mathbf{n}}\right)} \tan (d \sqrt{\mu})}\right)$.

in accordance with (13b). Substituting (16) and (20) along with (10) into Eq. (15), clearing the denominator, setting the coefficients of $\left(\frac{\psi^{\prime}}{\psi}\right)^{l}(l=0,2,4)$ to zero, we derive a system of nonlinear algebraic equations for $a_{0}, a_{1}, d, k$ and $\mu$. Solving the resulting system, we get the relation

$a_{0}=a_{0}, \quad a_{1}=\frac{\tan (d \sqrt{\mu})}{2 \sqrt{\mu}}, \quad k=-\frac{\sin (2 d \sqrt{\mu})}{\sqrt{\mu}}$,

which gives a discrete trigonometric function solution to Eq. (2) as $u_{n}(t)=a_{0}+\frac{1}{2}$

$$
\times \tan (d \sqrt{\mu})\left(\frac{-C_{1} \sin \left(\sqrt{\mu} \xi_{n}\right)+C_{2} \cos \left(\sqrt{\mu} \xi_{n}\right)}{C_{1} \cos \left(\sqrt{\mu} \xi_{n}\right)+C_{2} \sin \left(\sqrt{\mu} \xi_{n}\right)}\right),
$$

where $\xi_{n}=d n-\frac{\sin (2 d \sqrt{\mu})}{\sqrt{\mu}} t+\alpha$, while $a_{0}, d, \alpha, \mu(>0), C_{1}$ and $C_{2}$ remain arbitrary.

\subsection{Rational function solutions}

In case $\mu=0$, we first derive the expressions

$U_{n \pm 1}=a_{0}+a_{1}\left(\frac{\frac{\psi^{\prime}\left(\xi_{\mathbf{n}}\right)}{\psi\left(\xi_{\mathbf{n}}\right)}}{1 \pm d \frac{\psi^{\prime}\left(\xi_{\mathbf{n}}\right)}{\psi\left(\xi_{\mathbf{n}}\right)}}\right)$.

in accordance with (13c). Substituting (16) and (23) along with (10) into Eq. (15), clearing the denominator, setting the coefficients of $\left(\frac{\psi^{\prime}}{\psi}\right)^{l}(l=2,4)$ to zero, we derive a system of nonlinear algebraic equations for $a_{0}, a_{1}, d$ and $k$. Solving the resulting system, we get the relation

$a_{0}=a_{0}, \quad a_{1}=\frac{d}{2}, \quad k=-2 d$, which yields a discrete rational function solution to Eq. (2) as

$u_{n}(t)=a_{0}+\frac{d}{2}\left(\frac{C_{1}}{C_{1}(d n-2 d t+\alpha)+C_{2}}\right)$,

where $a_{0}, d, \alpha, C_{1}$ and $C_{2}$ remain arbitrary.

\subsection{Some special solutions}

We can further analyze our results by assigning special values to the arbitrary parameters $C_{1}$ and $C_{2}$. For example, if we set " $C_{1}=0$ and $C_{2} \neq 0$ " or " $C_{1} \neq 0$ and $C_{2}=0$ " in (19), respectively, then we get formal discrete solitary wave solutions to Eq. (2) as

$$
\begin{aligned}
& u_{n}(t)=a_{0}+\frac{1}{2} \tanh (d \sqrt{-\mu}) \tanh \left(\sqrt{-\mu}\left(d n-\frac{\sinh (2 d \sqrt{-\mu})}{\sqrt{-\mu}} t+\alpha\right)\right), \\
& u_{n}(t)=a_{0}+\frac{1}{2} \tanh (d \sqrt{-\mu}) \operatorname{coth}\left(\sqrt{-\mu}\left(d n-\frac{\sinh (2 d \sqrt{-\mu})}{\sqrt{-\mu}} t+\alpha\right)\right),
\end{aligned}
$$

where $a_{0}, d, \alpha$ and $\mu(<0)$ remain arbitrary (see Fig. 1 ).

By the same token, if we let " $C_{1} \neq 0$ and $C_{2}=0$ " or " $C_{1}=0$ and $C_{2} \neq 0$ " in (22) respectively, then we get formal discrete periodic wave solutions to Eq. (2) as

$u_{n}(t)=a_{0}-\frac{1}{2} \tan (d \sqrt{\mu}) \tan \left(\sqrt{\mu}\left(d n-\frac{\sin (2 d \sqrt{\mu})}{\sqrt{\mu}} t+\alpha\right)\right)$,
$u_{n}(t)=a_{0}+\frac{1}{2} \tan (d \sqrt{\mu}) \cot \left(\sqrt{\mu}\left(d n-\frac{\sin (2 d \sqrt{\mu})}{\sqrt{\mu}} t+\alpha\right)\right)$,

where $a_{0}, d, \alpha$ and $\mu(>0)$ remain arbitrary (see Fig. 2).

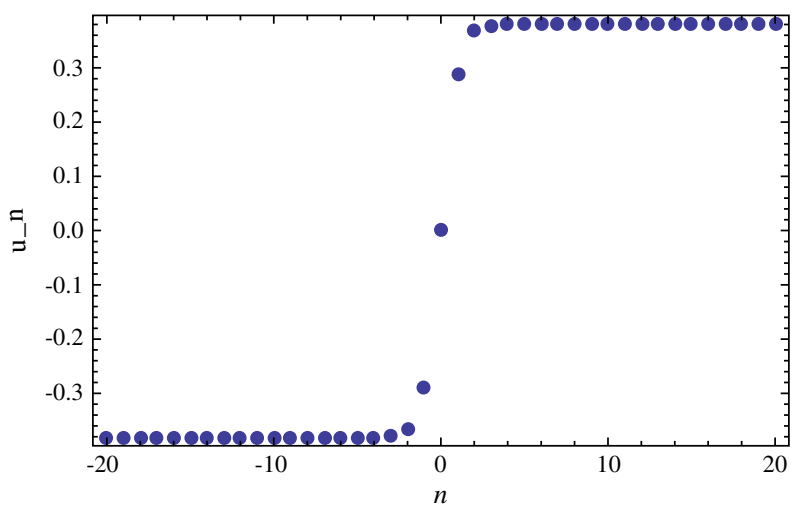

Fig. 1. A profile of (26) for $a_{0}=0, d=1, \mu=-1, \alpha=0$ at time $t=0$ for $n$ from -20 to 20.

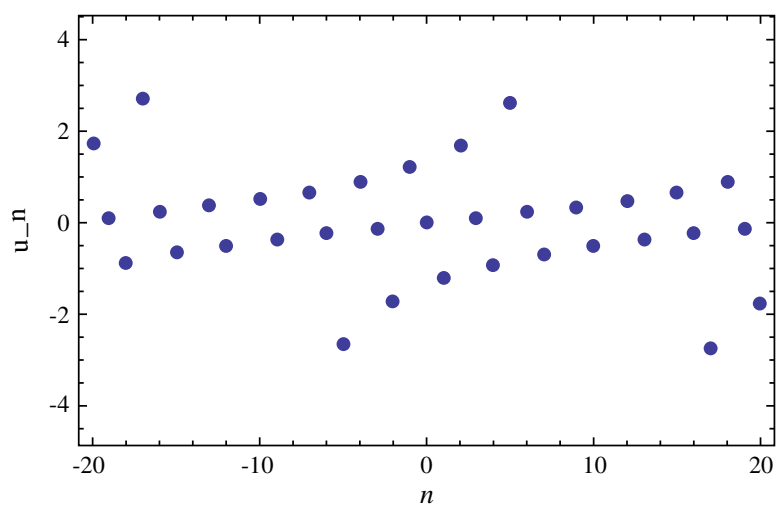

Fig. 2. A profile of (28) for $a_{0}=0, d=1, \mu=1, \alpha=0$ at time $t=0$ for $n$ from -20 to 20 


\section{Exact solutions for the system (3)}

Now, we aim to solve a system of fractional-type DDEs. As before, we first make the transformation

$u_{n}=U_{n}\left(\xi_{n}\right), \quad v_{n}=V_{n}\left(\xi_{n}\right), \quad \xi_{n}=d n+k t+\alpha$,

where $d$ and $k$ are real parameters to be specified, while $\alpha$ denotes the phase shift. Substituting (30) into the system (3) leads to the system

$k U_{n}^{\prime}=\frac{\left(V_{n+1}-V_{n-1}\right)\left(1-U_{n}^{2}\right)\left(1-V_{n}^{2}\right)}{\left(V_{n-1}+V_{n}\right)\left(V_{n}+V_{n+1}\right)}$,

$k V_{n}^{\prime}=\frac{\left(U_{n+1}-U_{n-1}\right)\left(1-U_{n}^{2}\right)\left(1-V_{n}^{2}\right)}{\left(U_{n-1}+U_{n}\right)\left(U_{n}+U_{n+1}\right)}$,

where prime denotes derivative with respect to the new independent variable $\xi_{n}$. Our procedure suggests then to look for special solutions of the system (31) in the form

$U_{n}=a_{0}+a_{1}\left(\frac{\psi^{\prime}\left(\xi_{n}\right)}{\psi\left(\xi_{n}\right)}\right), \quad a_{1} \neq 0$,

$V_{n}=b_{0}+b_{1}\left(\frac{\psi^{\prime}\left(\xi_{n}\right)}{\psi\left(\xi_{n}\right)}\right), \quad b_{1} \neq 0$,

where $\psi\left(\xi_{n}\right)$ satisfies Eq. (10), while $a_{0}, a_{1}, b_{0}$ and $b_{1}$ are arbitrary constants to be specified. Since the procedure is similar, it will be logical, from the next section on, to omit some details for brevity.

\subsection{Hyperbolic function solutions}

In case $\mu<0$, substituting (32), $U_{n \pm 1}$ and $V_{n \pm 1}$ along with (10) into the system (31), clearing the denominator, setting the coefficients of $\left(\frac{\psi^{\prime}}{\psi}\right)^{l}(l=0,1, \ldots, 6)$ to zero, we derive a system of nonlinear algebraic equations for $a_{0}, a_{1}, b_{0}, b_{1}, d, k$ and $\mu$. Solving the resulting system, we get the relations

$a_{0}=\mp \sqrt{1-2 b_{1} \sqrt{-\mu} \operatorname{coth}(d \sqrt{-\mu})-\mu b_{1}^{2}} /\left(1-2 b_{1} \sqrt{-\mu} \operatorname{coth}(d \sqrt{-\mu})\right)$,

$a_{1}=b_{1} /\left(1-2 b_{1} \sqrt{-\mu} \operatorname{coth}(d \sqrt{-\mu})\right)$,

$b_{0}=\mp \sqrt{1-2 b_{1} \sqrt{-\mu} \operatorname{coth}(d \sqrt{-\mu}) b_{1}-\mu b_{1}^{2}}$,

$b_{1}=b_{1}, k=-2 b_{1}^{2} \sqrt{-\mu} /\left(\tanh (d \sqrt{-\mu})-2 b_{1} \sqrt{-\mu}\right)$;

$a_{0}=\mp \sqrt{1-2 b_{1} \sqrt{-\mu} \operatorname{coth}(d \sqrt{-\mu})-\mu b_{1}^{2}} /\left(1-2 b_{1} \sqrt{-\mu} \operatorname{coth}(d \sqrt{-\mu})\right)$,

$a_{1}=-b_{1} /\left(1-2 b_{1} \sqrt{-\mu} \operatorname{coth}(d \sqrt{-\mu})\right)$,

$b_{0}= \pm \sqrt{1-2 b_{1} \sqrt{-\mu} \operatorname{coth}(d \sqrt{-\mu}) b_{1}-\mu b_{1}^{2}}, b_{1}=b_{1}$,

$k=2 b_{1}^{2} \sqrt{-\mu} /\left(\tanh (d \sqrt{-\mu})-2 b_{1} \sqrt{-\mu}\right)$

$a_{0}=\mp \sqrt{1+2 b_{1} \sqrt{-\mu} \operatorname{coth}(d \sqrt{-\mu})-\mu b_{1}^{2}} /\left(1+2 b_{1} \sqrt{-\mu} \operatorname{coth}(d \sqrt{-\mu})\right)$,

$a_{1}=b_{1} /\left(1+2 b_{1} \sqrt{-\mu} \operatorname{coth}(d \sqrt{-\mu})\right)$,

$b_{0}=\mp \sqrt{1+2 b_{1} \sqrt{-\mu} \operatorname{coth}(d \sqrt{-\mu})-\mu b_{1}^{2}}, b_{1}=b_{1}$,

$k=-2 b_{1}^{2} \sqrt{-\mu} /\left(\tanh (d \sqrt{-\mu})+2 b_{1} \sqrt{-\mu}\right)$;

$a_{0}=\mp \sqrt{1+2 b_{1} \sqrt{-\mu} \operatorname{coth}(d \sqrt{-\mu})-\mu b_{1}^{2}} /\left(1+2 b_{1} \sqrt{-\mu} \operatorname{coth}(d \sqrt{-\mu})\right)$,

$a_{1}=-b_{1} /\left(1+2 b_{1} \sqrt{-\mu} \operatorname{coth}(d \sqrt{-\mu})\right)$,

$b_{0}= \pm \sqrt{1+2 b_{1} \sqrt{-\mu} \operatorname{coth}(d \sqrt{-\mu})-\mu b_{1}^{2}}, b_{1}=b_{1}$,

$k=2 b_{1}^{2} \sqrt{-\mu} /\left(\tanh (d \sqrt{-\mu})+2 b_{1} \sqrt{-\mu}\right)$.
Here and henceforth, the signs are ordered vertically. Setting the parameter values (33)-(36) into the expression (32) in accordance with (11a), one can construct various types of discrete hyperbolic function solutions to the system (3). For instance, (33) leads to

$u_{n}(t)=\mp \frac{\sqrt{1-2 b_{1} \sqrt{-\mu} \operatorname{coth}(d \sqrt{-\mu})-\mu b_{1}^{2}}}{1-2 b_{1} \sqrt{-\mu} \operatorname{coth}(d \sqrt{-\mu})}$

$$
+\frac{b_{1} \sqrt{-\mu}}{1-2 b_{1} \sqrt{-\mu} \operatorname{coth}(d \sqrt{-\mu})}\left(\frac{C_{1} \cosh \left(\sqrt{-\mu} \xi_{\mathbf{n}}\right)+C_{2} \sinh \left(\sqrt{-\mu} \xi_{\mathbf{n}}\right)}{C_{1} \sinh \left(\sqrt{-\mu} \xi_{\mathbf{n}}\right)+C_{2} \cosh \left(\sqrt{-\mu} \xi_{\mathbf{n}}\right)}\right),
$$

$v_{n}(t)=\mp \sqrt{1-2 b_{1} \sqrt{-\mu} \operatorname{coth}(d \sqrt{-\mu}) b_{1}-\mu b_{1}^{2}}$

$+b_{1} \sqrt{-\mu}\left(\frac{C_{1} \cosh \left(\sqrt{-\mu} \xi_{\mathbf{n}}\right)+C_{2} \sinh \left(\sqrt{-\mu} \xi_{\mathbf{n}}\right)}{C_{1} \sinh \left(\sqrt{-\mu} \xi_{\mathbf{n}}\right)+C_{2} \cosh \left(\sqrt{-\mu} \xi_{\mathbf{n}}\right)}\right)$

where $\xi_{n}=d n-\frac{2 b_{1}^{2} \sqrt{-\mu}}{\tanh (d \sqrt{-\mu})-2 b_{1} \sqrt{-\mu}} t+\alpha$, while $b_{1}, d, \alpha, \mu(<0), C_{1}$ and $C_{2}$ remain arbitrary.

\subsection{Trigonometric function solutions}

In case $\mu>0$, substituting (32), $U_{n \pm 1}$ and $V_{n \pm 1}$ along with (10) into the system (31), clearing the denominator, setting the coefficients of $\left(\frac{\psi^{\prime}}{\psi}\right)^{l}(l=0,1, \ldots, 6)$ to zero, we derive a system of nonlinear algebraic equations for $a_{0}, a_{1}, b_{0}, b_{1}, d, k$ and $\mu$. Solving the resulting system, we get the relations

$$
\begin{aligned}
& a_{0}=\mp \sqrt{1-2 b_{1} \sqrt{\mu} \cot (d \sqrt{\mu})-\mu b_{1}^{2}} /\left(1-2 b_{1} \sqrt{\mu} \cot (d \sqrt{\mu})\right), \\
& a_{1}=-b_{1} /\left(1-2 b_{1} \sqrt{\mu} \cot (d \sqrt{\mu})\right) \\
& b_{0}= \pm \sqrt{1-2 b_{1} \sqrt{\mu} \cot (d \sqrt{\mu})-\mu b_{1}^{2}}, \quad b_{1}=b_{1}, \\
& k=2 b_{1}^{2} \sqrt{\mu} /\left(\tan (d \sqrt{\mu})-2 b_{1} \sqrt{\mu}\right) ;
\end{aligned}
$$

$$
\begin{aligned}
& a_{0}=\mp \sqrt{1-2 b_{1} \sqrt{\mu} \cot (d \sqrt{\mu})-\mu b_{1}^{2}} /\left(1-2 b_{1} \sqrt{\mu} \cot (d \sqrt{\mu})\right), \\
& a_{1}=b_{1} /\left(1-2 b_{1} \sqrt{\mu} \cot (d \sqrt{\mu})\right) \\
& b_{0}=\mp \sqrt{1-2 b_{1} \sqrt{\mu} \cot (d \sqrt{\mu})-\mu b_{1}^{2}}, \quad b_{1}=b_{1}, \\
& k=-2 b_{1}^{2} \sqrt{\mu} /\left(\tan (d \sqrt{\mu})-2 b_{1} \sqrt{\mu}\right) ;
\end{aligned}
$$

$$
\begin{aligned}
& a_{0}=\mp \sqrt{1+2 b_{1} \sqrt{\mu} \cot (d \sqrt{\mu})-\mu b_{1}^{2}} /\left(1+2 b_{1} \sqrt{\mu} \cot (d \sqrt{\mu})\right), \\
& a_{1}=b_{1} /\left(1+2 b_{1} \sqrt{\mu} \cot (d \sqrt{\mu})\right), \\
& b_{0}=\mp \sqrt{1+2 b_{1} \sqrt{\mu} \cot (d \sqrt{\mu})-\mu b_{1}^{2}}, \quad b_{1}=b_{1}, \\
& k=-2 b_{1}^{2} \sqrt{\mu} /\left(\tan (d \sqrt{\mu})+2 b_{1} \sqrt{\mu}\right) ;
\end{aligned}
$$

$$
\begin{aligned}
& a_{0}=\mp \sqrt{1+2 b_{1} \sqrt{\mu} \cot (d \sqrt{\mu})-\mu b_{1}^{2}} /\left(1+2 b_{1} \sqrt{\mu} \cot (d \sqrt{\mu})\right), \\
& a_{1}=-b_{1} /\left(1+2 b_{1} \sqrt{\mu} \cot (d \sqrt{\mu})\right), \\
& b_{0}= \pm \sqrt{1+2 b_{1} \sqrt{\mu} \cot (d \sqrt{\mu})-\mu b_{1}^{2}}, \quad b_{1}=b_{1}, \\
& k=2 b_{1}^{2} \sqrt{\mu} /\left(\tan (d \sqrt{\mu})+2 b_{1} \sqrt{\mu}\right) .
\end{aligned}
$$

Setting the parameter values (38)-(41) into the expression (32) in accordance with (11b), one can construct various types of discrete trigonometric function solutions to the system (3). For example, (38) gives 


$$
\begin{aligned}
u_{n}(t)= & \mp \frac{\sqrt{1-2 b_{1} \sqrt{\mu} \cot (d \sqrt{\mu})-\mu b_{1}^{2}}}{\left(1-2 b_{1} \sqrt{\mu} \cot (d \sqrt{\mu})\right)}-\frac{b_{1} \sqrt{\mu}}{\left(1-2 b_{1} \sqrt{\mu} \cot (d \sqrt{\mu})\right)} \\
& \times\left(\frac{-C_{1} \sin \left(\sqrt{\mu} \xi_{\mathrm{n}}\right)+C_{2} \cos \left(\sqrt{\mu} \xi_{\mathrm{n}}\right)}{C_{1} \cos \left(\sqrt{\mu} \xi_{\mathrm{n}}\right)+C_{2} \sin \left(\sqrt{\mu} \xi_{\mathrm{n}}\right)}\right), \\
v_{n}(t)= & \pm \sqrt{1-2 b_{1} \sqrt{\mu} \cot (d \sqrt{\mu})-\mu b_{1}^{2}} \\
& +b_{1} \sqrt{\mu}\left(\frac{-C_{1} \sin \left(\sqrt{\mu} \xi_{\mathrm{n}}\right)+C_{2} \cos \left(\sqrt{\mu} \xi_{\mathrm{n}}\right)}{C_{1} \cos \left(\sqrt{\mu} \xi_{\mathrm{n}}\right)+C_{2} \sin \left(\sqrt{\mu} \xi_{\mathrm{n}}\right)}\right),
\end{aligned}
$$

where $\xi_{n}=d n+\frac{2 b_{1}^{2} \sqrt{\mu}}{\tan (d \sqrt{\mu})-2 b_{1 \sqrt{\mu}}} t+\alpha$, while $b_{1}, d, \alpha, \mu(>0), C_{1}$ and $C_{2}$ remain arbitrary.

\subsection{Rational function solutions}

In case $\mu=0$, substituting (32), $U_{n \pm 1}$ and $V_{n \pm 1}$ along with (10) into the system (31), clearing the denominator, setting the coefficients of $\left(\frac{\psi^{\prime}}{\psi}\right)^{l}(l=0,1, \ldots, 6)$ to zero, we derive a system of nonlinear algebraic equations for $a_{0}, a_{1}, b_{0}, b_{1}, d$ and $k$. Solving the resulting system, we get the relations

$$
\begin{aligned}
& a_{0}=\mp \frac{d}{d-2 b_{1}} \sqrt{1-\frac{2 b_{1}}{d}}, \quad a_{1}=-\frac{d b_{1}}{d-2 b_{1}}, \\
& b_{0}= \pm \sqrt{1-\frac{2 b_{1}}{d}}, \quad b_{1}=b_{1}, \quad k=\frac{2 b_{1}^{2}}{d-2 b_{1}}, \\
& a_{0}=\mp \frac{d}{d-2 b_{1}} \sqrt{1-\frac{2 b_{1}}{d}}, \quad a_{1}=\frac{d b_{1}}{d-2 b_{1}}, \\
& b_{0}=\mp \sqrt{1-\frac{2 b_{1}}{d}}, \quad b_{1}=b_{1}, \quad k=-\frac{2 b_{1}^{2}}{d-2 b_{1}}, \\
& a_{0}=\mp \frac{d}{d+2 b_{1}} \sqrt{1+\frac{2 b_{1}}{d}}, \quad a_{1}=\frac{d b_{1}}{d+2 b_{1}}, \\
& b_{0}=\mp \sqrt{1+\frac{2 b_{1}}{d}}, \quad b_{1}=b_{1}, \quad k=-\frac{2 b_{1}^{2}}{d+2 b_{1}}, \\
& a_{0}=\mp \frac{d}{d+2 b_{1}} \sqrt{1+\frac{2 b_{1}}{d}}, \quad a_{1}=-\frac{d b_{1}}{d+2 b_{1}}, \\
& b_{0}= \pm \sqrt{1+\frac{2 b_{1}}{d}}, \quad b_{1}=b_{1}, \quad k=\frac{2 b_{1}^{2}}{d+2 b_{1}} .
\end{aligned}
$$

Inserting the parameter values (43)-(46) into the expression (32) in accordance with (11c), it is possible construct various types of discrete rational function solutions to the system (3). For instance, (43) yields

$$
\begin{aligned}
& u_{n}(t)=\mp \frac{d}{d-2 b_{1}} \sqrt{1-\frac{2 b_{1}}{d}}-\frac{d b_{1}}{d-2 b_{1}}\left(\frac{C_{1}}{C_{1}\left(d n+\frac{2 b_{1}^{2}}{d-2 b_{1}} t+\alpha\right)+C_{2}}\right), \\
& v_{n}(t)= \pm \sqrt{1-\frac{2 b_{1}}{d}}+b_{1}\left(\frac{C_{1}}{C_{1}\left(d n+\frac{2 b_{1}^{2}}{d-2 b_{1}} t+\alpha\right)+C_{2}}\right),
\end{aligned}
$$

where $b_{1}, d, \alpha, C_{1}$ and $C_{2}$ remain arbitrary. Of course, as was done in the preceding section, one can assign special values to the arbitrary parameters $C_{1}$ and $C_{2}$ in the above expressions for further analysis. We skip this procedure for the sake of brevity.

Remark 1. In exact solution methods, the reduction of given PDEs to simpler integrable ODEs is quite common. The essential part of our method, which can be thought as a special case of the transformed rational function method [23], is based on the proposal that the special functions that one takes to expand the exact solution are the general solution of simpler ODE with a higher order than the original differential equation with eminent solution. The main point of the transformed rational function method, which successfully unifies some already known methods, is to look for rational solutions to variable-coefficient ODEs deduced from given PDEs. This is accomplished with the introduction of a new variable by a solvable ODE. Another sophifisticated technique is the Frobenius integrable decompositions (FIDs) method [24] for PDEs. The core point of FIDs method is to transform nonlinear PDEs into systems of Frobenius integrable ODEs with cubic nonlinearity. The invariant subspace method [25] is also introduced to demonstrate more unity and more diversity of exact solutions to PDEs. The key idea is to take subspaces of solutions to linear ODEs as invariant subspaces that PDEs assume. The crucial point of the ansatze method [26] is to choose a simplest equation which is solvable by quadratures. For instance, using the general Ricatti equation $v_{\xi}=a_{0}+a_{1} v+a_{2} v^{2}, a_{2} \neq 0$, more general solutions to PDEs could be generated. We refer the formulas (40)(42) in [26] for the solutions of the general Ricatti equation. The discrete Jacobi sub-equation method presented in [27] provides exact solutions to nonlinear DDEs in a unified form by means of Jacobi elliptic functions $s n, c n$ and $d n$. The essential idea of the method is to reduce the difference terms of DDEs by an auxiliary difference equation and the differential terms of DDEs by an auxiliary differential equation.

\section{Conclusion}

In general, it is known that fractional-type DDEs are difficult to tackle. Nevertheless, we have shown that some can be solved by the extended simplest equation method in a straightforward way. For the equations under study, three types of exact solutions (hyperbolic, trigonometric and rational) are computed for the first time. Some solutions are analyzed by assigning special values to the parameters. We checked the correctness of the solutions by putting them back into the original equation. This provides an extra measure of confidence in the results. The solution procedure of our method is easy, reliable and efficient, as well as does not require a large amount of run-time with the help of a computer algebra system like MATHEMATICA. Of course, we are aware of the fact that not all such equations can be treated with this method. For example, our approach does not yield any real-valued solutions to the following fractional-type DDE [12]

$\dot{u}_{n}=\frac{\left(u_{n-1}-u_{n+1}\right)\left(\alpha u_{n}^{4}+\beta u_{n}^{2}+\gamma\right)}{\left(U_{n-1}+U_{n}\right)\left(U_{n}+U_{n+1}\right)}$,

where $\alpha, \beta$ and $\gamma$ are arbitrary parameters. However, we believe that we achieved our goal of providing exact and explicit solutions for the Eq. (2) and the system (3) which are subject to some adequate physical interpretations in the future. In conclusion, it seems that the extended simplest equation method can be used for several other fractional-type DDEs. This will be our next step to be undertaken later on.

\section{Acknowledgment}

The author is grateful to the anonymous referee for the suggestion of some useful references.

\section{References}

[1] Fermi E, Pasta J, Ulam S. Collected Papers of Enrico Fermi. Chicago: University of Chicago Press; 1965. 
[2] Wadati M. Transformation theories for nonlinear discrete systems. Prog Suppl Theor Phys 1976;59:36-63.

[3] Ohta Y, Hirota R. A discrete KdV equation and its Casorati determinant solution. J Phys Soc Jpn 1991;60:2095

[4] Toda M. Theory of nonlinear lattices. New-York: Springer-Verlag; 1989.

[5] Ablowitz MJ, Ladik J. Nonlinear differential-difference equation. J Math Phys 1975;16:598-603.

[6] Orfandis SJ. Sine-Gordon equation and nonlinear $\sigma$ model on a lattice. Phys Rev D 1978;18:3828-32.

[7] Hirota R. Exact $N$-soliton solution of nonlinear lumped self-dual network equations. J Phys Soc Jpn 1973;35:289-94.

[8] Baldwin D, Göktaş Ü, Hereman W. Symbolic computation of hyperbolic tangent solutions for nonlinear differential-difference equations. Comput Phys Commun 2004;162:203-17.

[9] Capel HW, Wiersma GL. Linearizing integral transform for the multicomponent lattice KP. Physica A 1986;138:76-99.

[10] Wiersma GL, Capel HW. Lattice equations, hierarchies and Hamiltonian structures. Physica A 1987;142:199-244.

[11] Narita K. New discrete modified KdV equation. Progr Theoret Phys 1991;86:817-24.

[12] Narita K. Solutions for a system of difference-differential equations related to the self-dual network equation. Progr Theoret Phys 2001;106:1079-96.

[13] Hirota R, Satsuma J. A variety of nonlinear network equations generated from the Bäcklund transformation for the Toda lattice. Progr Theoret Phys Suppl 1976;59:64-100.

[14] Noguchi A, Watanabe H, Sakai K. Shock wave and hole type soliton of nonlinear self-dual network equation. J Phys Soc Jpn 1977;43: 1441-6.
[15] Noguchi A, Watanabe H, Sakai K. Solitons of the nonlinear self-dual network with saturable property. J Phys Soc Jpn 1978;45:2009-13.

[16] Hu XB, Ma WX. Application of Hirota's bilinear formalism to the Toeplitz lattice-some special soliton-like solutions. Phys Lett A 2002;293:161-5.

[17] Yang P, Chen Y, Li ZB. ADM-Padé technique for the nonlinear lattice equations. Appl Math Comput 2009;210:362-75.

[18] Ma WX, You Y. Rational solutions of the Toda lattice equation in Casoratian form. Chaos Solitons Fract 2004;22:395-406.

[19] Zhu SD, Chu YM, Qiu SL. The homotopy perturbation method for discontinued problems arising in nanotechnology. Comput Math Appl 2009;58:2398-401.

[20] Dai CQ Wang YY. Exact traveling wave solutions of the discrete nonlinear Schrödinger equation and the hybrid lattice equation obtained via the expfunction method. Phys Scr 2008;78:015013.

[21] Kudryashov NA, Loguinova NB. Extended simplest equation method for nonlinear differential equations. Appl Math Comput 2008;205:396-402.

[22] Aslan İ. A discrete generalization of the extended simplest equation method. Commun Nonlinear Sci Numer Simul 2010;15:1967-73.

[23] Ma WX, Lee JH. A transformed rational function method and exact solutions to the $3+1$ dimensional Jimbo-Miwa equation. Chaos Solitons Fract 2009;42:1356-63.

[24] Ma WX, Wu H, He J. Partial differential equations possessing Frobenius integrable decompositions. Phys Lett A 2007;364:29-32.

[25] Ma WX. A refined invariant subspace method and applications to evolution equations. Sci China Math 2012;55:1769-78.

[26] Ma WX, Fuchssteiner B. Explicit and exact solutions to a KolmogorovPetrovskii-Piskunov equation. Int J Nonlinear Mech 1996;31:329-38.

[27] Wang Z, Ma WX. Discrete Jacobi sub-equation method for nonlinear differential-difference equations. Math Meth Appl Sci 2010;33:1463-72. 